Bull. Mater. Sci., Vol. 22, No. 3, May 1999, pp. 723-728. (C) Indian Academy of Sciences.

\title{
Creation and motion of dislocations and fracture in metal and silicon crystals
}

\author{
MASAO DOYAMA \\ Teikyo University of Science and Technology, Uenohara, Yamanashi 409-0193, Japan
}

\begin{abstract}
By making a step on one surface $(11 \overline{2})$ of a rectangular small paralellepiped copper crystal, dislocations could be created by the molecular dynamic method. The dislocation created was not a complete edge dislocation but a pair of Heidenreich-Shockley partial dislocations. Each time a dislocation was created, the stress on the surface was released. Small copper crystals having a notch were pulled (until fracture), compressed and buckled by use of the molecular dynamic method. An embedded atom potential was used to represent the interaction between atoms. Dislocations were created near the tip of the notch. A very sharp yield stress was observed.

The results of high speed deformations of pure silicon small crystals using the molecular dynamics are presented. The results suggest that plastic deformation may be possible for the silicon with a high speed deformation even at room temperature. Another small size single crystal, the same size and the same surfaces, was compressed using molecular dynamic method. The surfaces are $\{110\},\{112\}$ and $\{111\}$. The compressed direction was [111]. It was found that silicon crystals are possible to be compressed with a high speed deformation. This may suggest that silicon may be plastically deformed with high speed deformation.
\end{abstract}

Keywords. Simulation; creation of dislocations; tensile deformation; copper single crystals; silicon single crystals.

\section{Introduction}

Micromachining and nanomachining are becoming increasingly popular and it is important to clarify the micromechanism of plastic deformation and fracture of small crystals. It is well known that the plastic deformation of metallic crystals occurs by the creation, motion and cutting of dislocations. It is quite important to know where the dislocations are created during the deformation and how these dislocations move in specimens when they are deformed. In this paper, the molecular dynamics method is used to simulate the deformation of small single crystals. Pair potentials are often used to represent the interaction potentials between atoms, but in metals especially the effect of the surface is important and the pair potentials are not ideal to represent the interactions near the surface. Therefore, embedded atom potentials have been developed to overcome this difficulty. In this paper, the creation and motion of dislocations in copper crystals have been simulated by molecular dynamics with embedded atom potentials (Oh and Johnson 1986, 1989; Doyama and Kogure 1997).

\section{Creation of dislocations (Doyama and Yamamoto 1987)}

Specimen C-1 (figure 1a) is rectangular parallelepiped copper crystal having the faces of (111), ( $\overline{1} \overline{1} \overline{1})$; $(11 \overline{2})$, $(\overline{1} \overline{1} 2) ;(1 \overline{1} 0)$ and $(\overline{1} 10), x, y$, and $z$ directions were taken in the [1110], [ $\overline{1} \overline{1} 2]$ and [111] directions, respectively. Specimen $\mathrm{C}-2$ is similar to specimen $\mathrm{C}-1$ but with a notch on the (110). A step (height $=0.01 d$, where $d$ is the nearest neighbour distance) was made on the (110), then all the atoms in the crystal were relaxed 50 cycles by molecular dynamics. The step height was again increased by $0.01 \mathrm{~d}$. This process was repeated.

A Heidenreich-Schockley (H-S) partial dislocation was created followed by the other H-S partial dislocation. Immediately with the creation of the first dislocation, the stress was greatly reduced and a very sharp yield point was observed. Figure 2 shows the projection of atoms on the $(11 \overline{2})$ plane. Figure 3 shows the projection of atoms two layers above the slip plane and one layer below the slip plane on the (111) plane. Stacking faults are clearly seen. The stress-strain curves for specimens $\mathrm{C}-1$ and $\mathrm{C}-2$ are plotted in figure 4 . With each introduction of a half-dislocation, a sharp yield stress was observed. Two half-dislocations are connected with the stacking fault.

\section{Deformation and fracture of single crystals (Doyama 1997)}

Specimen C-3 (figure 1c) contains 4338 atoms having the faces of $(100),(\overline{1} 00) ;(010),(0 \overline{1} 0) ;(001)$ and $(00 \overline{1})$. The $x, y$, and $z$ directions were taken to be in the 


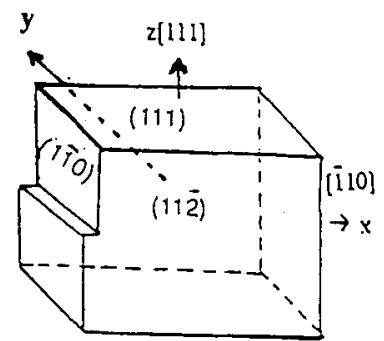

(a)

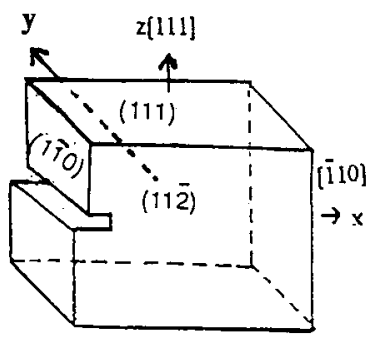

(b)

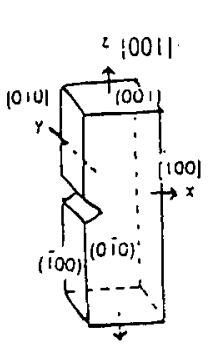

(c)

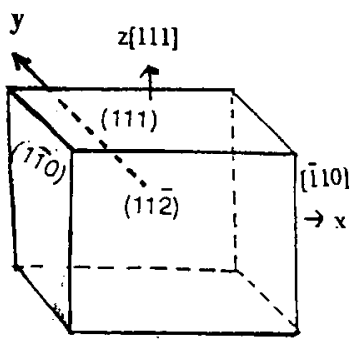

(d)

Figure 1. (a) Copper specimen C-1, (b) copper specimen C-2, (c) copper specimen C-3, (d) silicon specimen S-1.

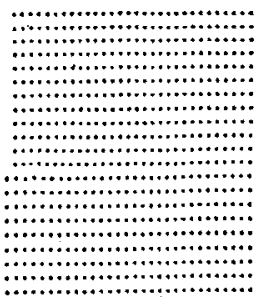

(a)

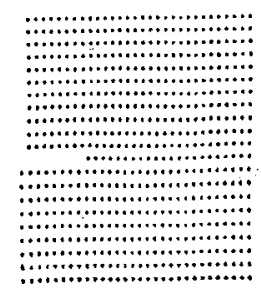

(d)

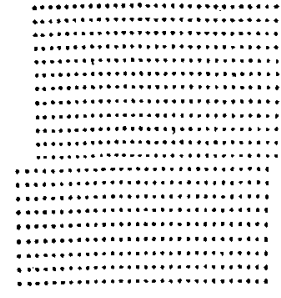

(b)

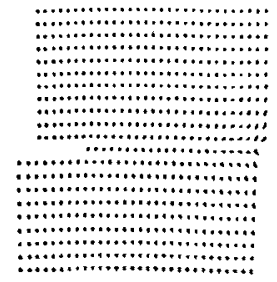

(e)

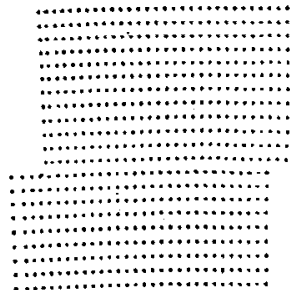

(c)

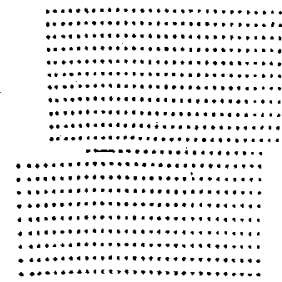

(f)

Figure 2. Projection of copper atoms on the $(11 \overline{2})$ plane of specimens C-1 and C-2. Creation of dislocations. (a), (b), and (c) are specimen C-1 without a notch and (d), (e) and (f) are for specimen $\mathrm{C}-2$ with a notch.

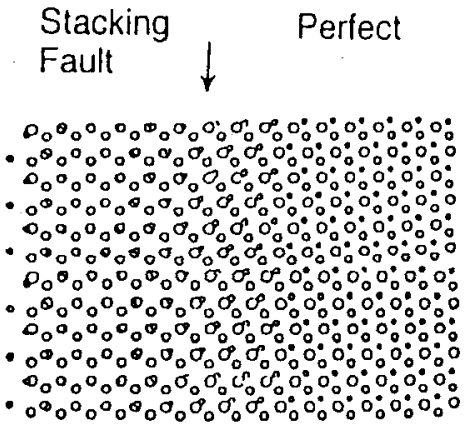

(a)
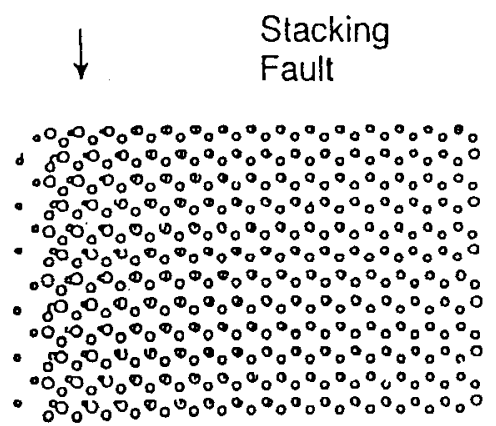

(b)
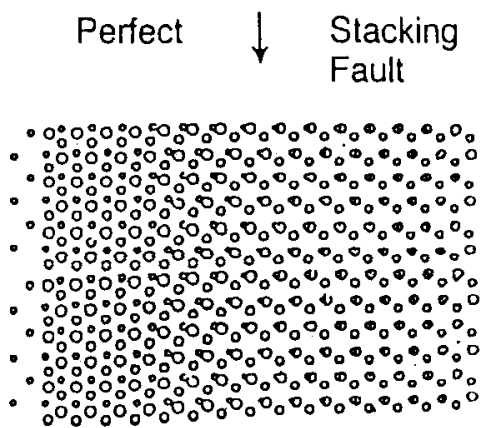

(c)

Figure 3. Projection of atoms in two layers above the slip plane and one layer below the slip plane on the (111) plane in specimen C-1. Two half-dislocations are connected with a stacking fault. (a) 3500 time steps (0.7d step height), (b) at 5000 time steps (1.0 $d$ step height) and (c) at 6000 time steps (1.2 $d$ step height). Arrows show half-dislocations. 
direction of [100], [010], and [001], respectively. The size of specimen C-3 was $8 a \times 8 a \times 26 a$, where $a$ is the lattice parameter and a notch was made in the $y$ direction at the centre on the $(\overline{100)}$. The surfaces were free and the periodic boundary conditions were not used. Specimen C-3 was pulled in the [001] direction by $0.5 \%$, i.e. all the atoms were deformed uniformly, then all atoms except the one layer of each end were relaxed 25 cycles, and again were pulled $0.5 \%$ in the [001] direction, and once again all the atoms were relaxed 25 cycles. The atoms in the end layers were relaxed only in the $x$ and $y$ directions and not in the $z$ direction. This procedure was repeated. The time step of the molecular dynamics was $5 \times 10^{-15} \mathrm{~s}$.

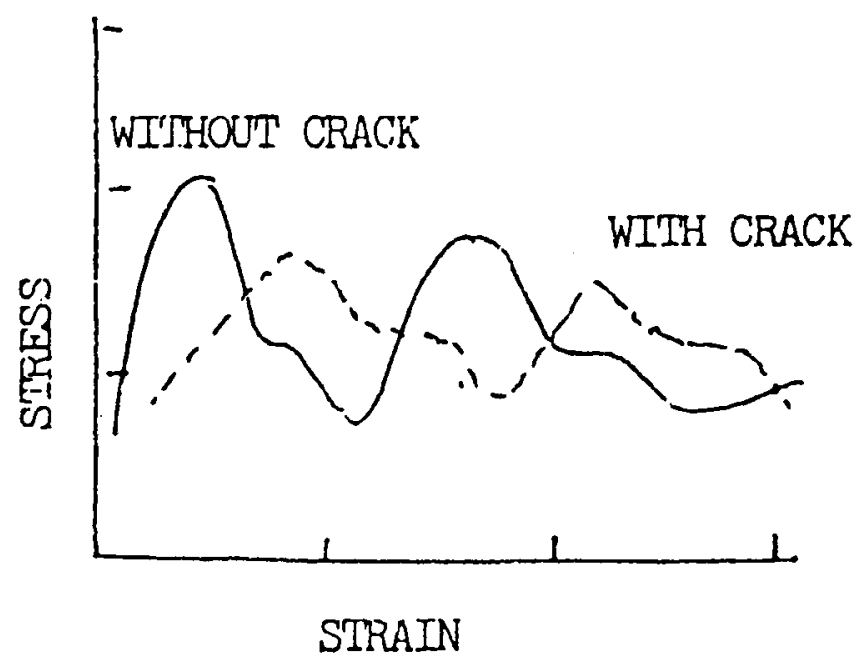

Figure 4. Stress-strain curves for specimens C-1 and C-2.
Atomic positions near the centre of specimen C-3 projected on the $x z$ plane $(010)$ are plotted in figures $5 a-d$. In figure $5 b$, near the tip of the crack, a halfdislocation was created. Two other half-dislocations are started near the grip of specimen C-3. These can be clearly seen when the reader looks at the figures at a small angle. In figure 6 the stress-strain curve is plotted. A very sharp yield point was found.

It was found that a notch is normally a source of dislocation due to the stress concentration. [2 111$]$ (111) and [1피] (111) half-dislocations were created leaving stacking faults. A schematic diagram is given in figure 7 for specimen C-3. The initial stage of deformation was clearly conducted by the creation and motion of dislocations. In the middle stage, dislocations on different slip planes cross each other. The work hardening occurs in this stage. In the final stage, in the case of copper, a crystalline state with many dislocations occurs and the inhomogeneous deformation is normal. Figure 8 shows the atomic positions just after fracture. A polycrystalline state near the fracture was observed.

\section{Cutting and compression of silicon single crystal}

Silicon is becoming a very important material in the field of electronics and communication. Device production process of silicon is also becoming important and the scale is becoming finer and finer. In these processes, micromachining is one of the important processes in the field of electronic devices. Cutting, and compression of single crystals have been simulated using molecular dynamics. In this paper silicon single crystals were cut by idealized tools. The results are compared with those of metals.

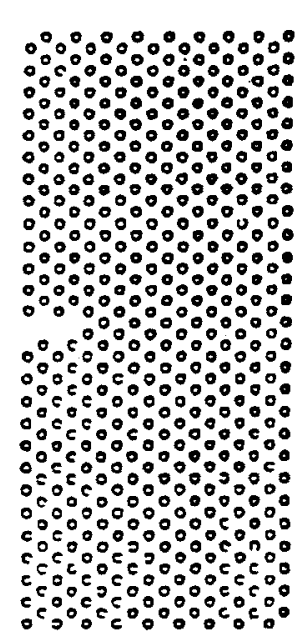

(a)

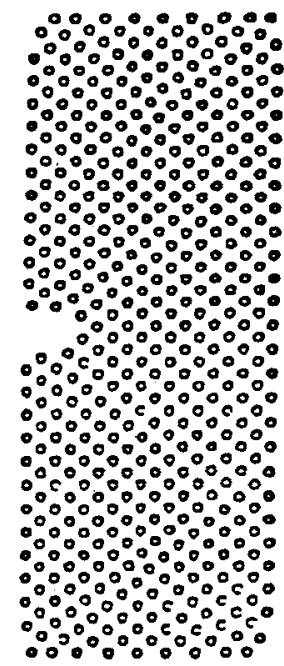

(b)

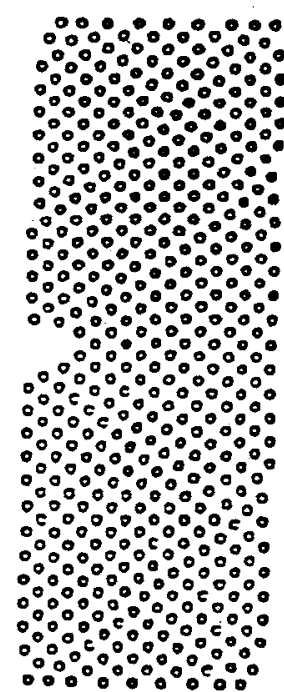

(c)

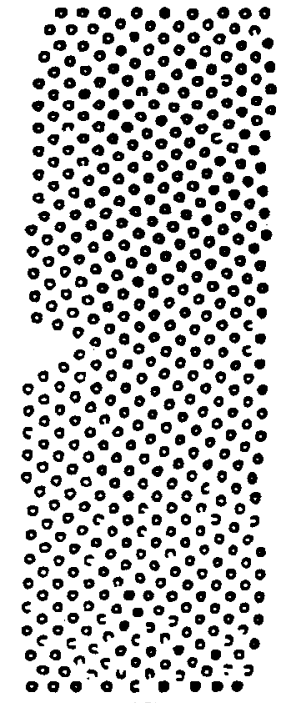

(d)

Figure 5. Specimen C-3 (a) 300 time steps $(6 \%$ elongation), (b) 375 time steps $(7.5 \%$ elongation), (c) 500 time steps (10\% elongation), and (d) 675 time steps (13.5\%). All are projected on $x z$ (010) plane. 
The potential used here is proposed by Stillinger and Weber $(1983,1984,1985)$. The specimen used was a three-dimensional single crystal having 1116 atoms surrounded by $(\overline{1} 10),(1 \overline{1} 0) ;(11 \overline{2}),(\overline{1} \overline{1} 2) ;(111)$, and $(\overline{1} \overline{1})$. The simulation of cutting a thin layer on (111) of silicon has been carried out. The size of a crystal is $6.0[x] \times 1.6[y] \times 2.0[z][\mathrm{nm}] . x, y$, and $z$ axes are taken in $[\overline{1} 10],[\overline{1} \overline{1} 2]$, and [111] directions, respectively. [110] direction is the cutting direction. (111) is a slip plane. A sharp solid edge shown was advanced in the $x$ direction with a speed of $10 \mathrm{~m} / \mathrm{s}$. Periodic boundary condition was not used. Surfaces were free. The time step interval was taken to be $1 \times 10^{-16} \mathrm{sec}$. The simulation
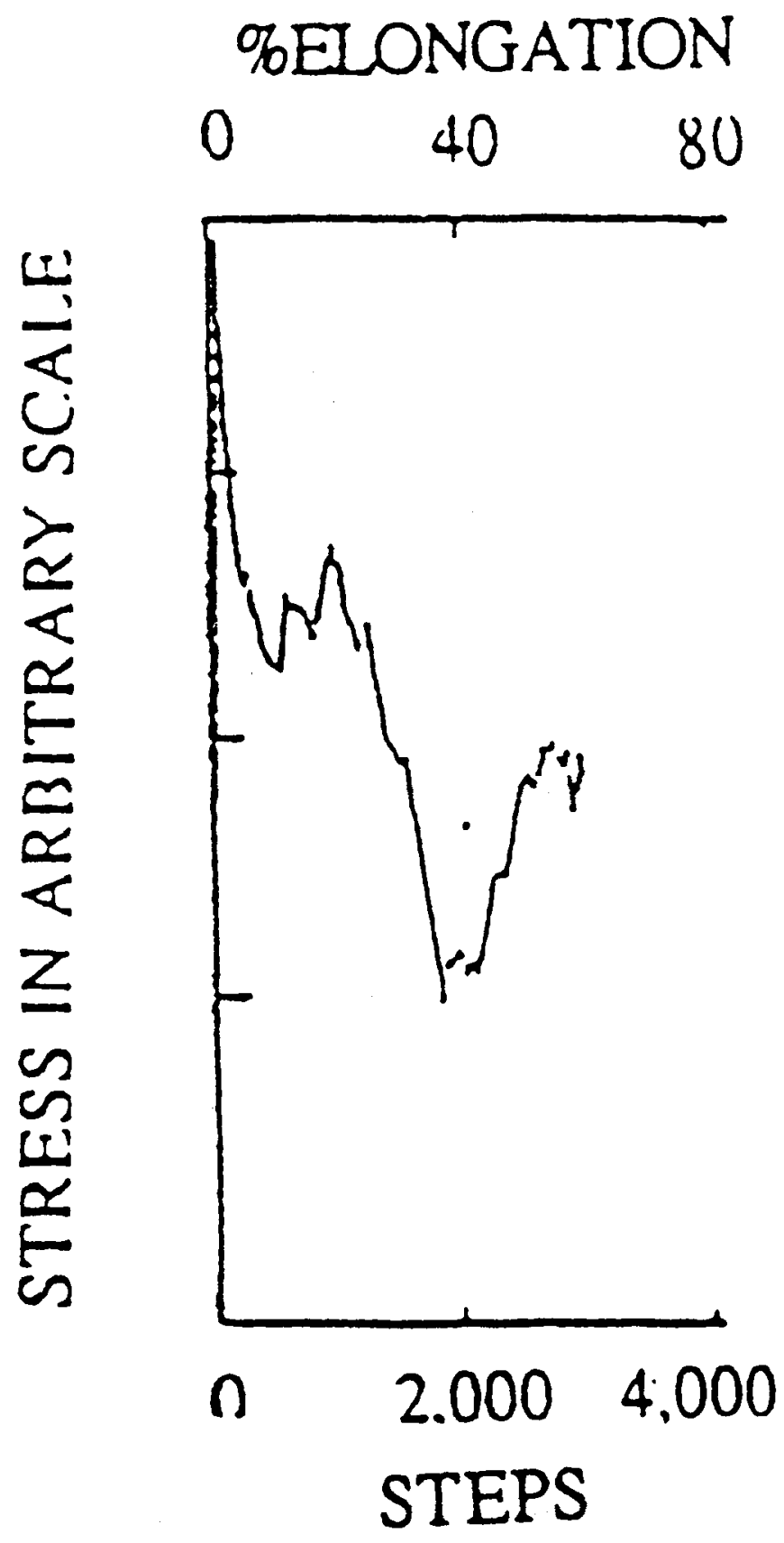

Figure 6. Stress-strain curve for specimen C-3.

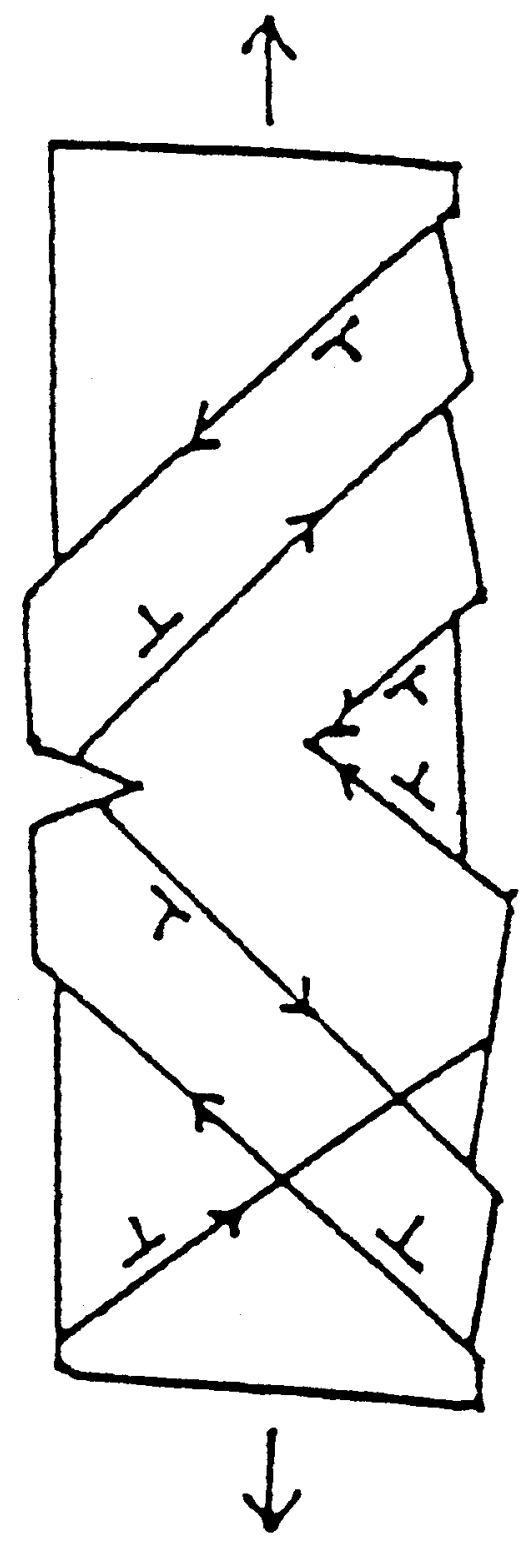

Figure 7. Schematic diagram of creation and motion of halfdislocation in specimen C-3.

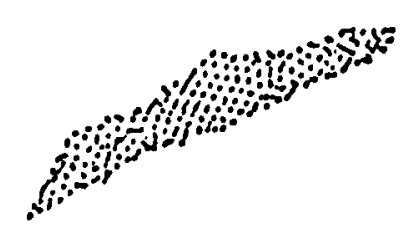

Figure 8. Atomic position just after fracture. 


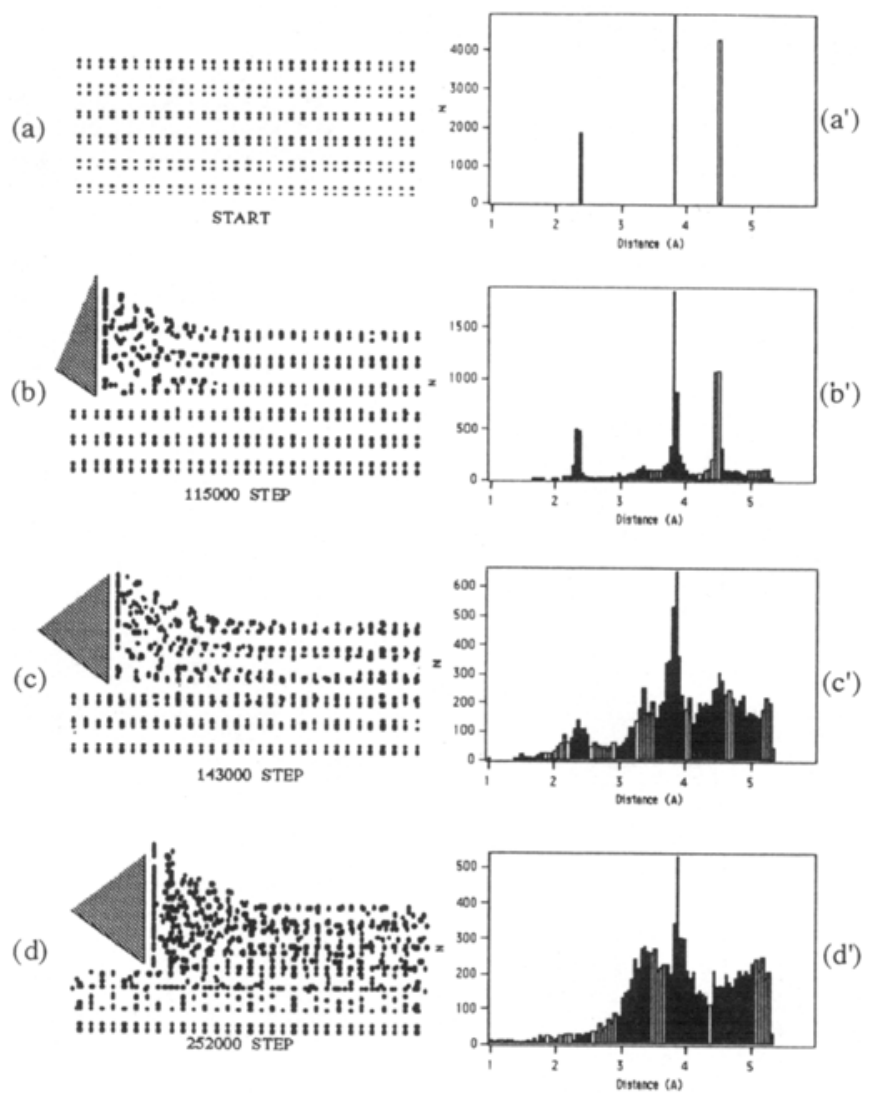

Figure 9. Atomic positions and radial distribution function of atoms near the tool. (a) start, (b) at 115,000 steps, (c) 143,000 steps, (d) 252,000 steps. (c) and (d) clearly show the region near the tool is amorphous.

was extended up to 400,000 steps. The thickness of the cut was set to 2, 4 and 6 atomic layers of silicon from the top of the crystal. Every 100 steps the kinetic energy was reduced to zero (quench). The position of atoms are projected to (112) plane and the radial distribution function at the start of cutting, 115,000, 143,000, and 252,000 steps, respectively are shown (figure 9). Atoms in front of the cutting edge were severely displaced. Near the cutting edge, atoms were pushed up. The chip became amorphous. Figures $9\left(c^{\prime}\right)$ and $\left(d^{\prime}\right)$ show the region to be amorphous. The creation of dislocations was not clearly observed, but the amorphous region was extended. Moreover the cut surface also became amorphous as the cutting proceeded. Figure 10 shows the projection of atomic positions on (112) during compression. The crystal also became amorphous.

- The cutting speeds were $10 \mathrm{~m} / \mathrm{s}-50 \mathrm{~m} / \mathrm{s}$. Chips became amorphous in all cases. The position near the cutting tool also became amorphous. The cut surface became also amorphous in the later stage. In case of copper, chips and specimen surface did not become amorphous but became polycrystalline. It was found that the cut surface also became amorphous. This was clearly shown by examining the radial distribution function of deformed atoms. The plastic deformation was restricted in a limited area. The surface was smoother when the cutting thickness was thicker. Atomic shuffling was not observed even though it was observed in the case of copper. The creation of dislocations was not observed, but the specimen became amorphous.

Silicon single crystals were found to be harder than those of metals. The plastic deformation was restricted in a limited area. The surface was smoother when the cutting thickness was thicker.

For compression, the specimen was the same as that for cutting. The crystal was compressed in the [111] direction. Silicon crystals can be plastically compressed by a ultra high speed compression by simulation.

Further study is necessary to understand plastic deformation of silicon.

\section{Conclusions}

Creation of dislocations and plastic deformation of copper and silicon were studied by use of molecular dynamics. In copper always Heidenreich-Shockley partial dislocations were created near the tip of the notch of copper. As soon as the partial dislocations were created 


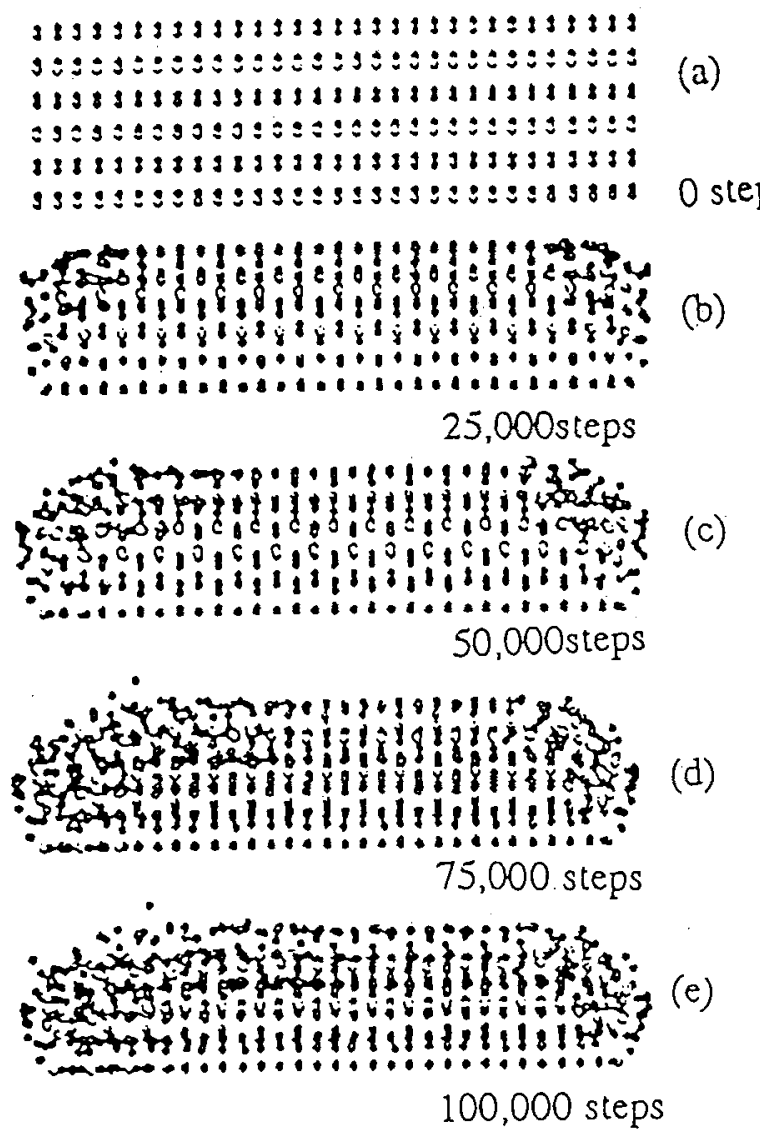

\section{(a)}

step

(b)

(g)

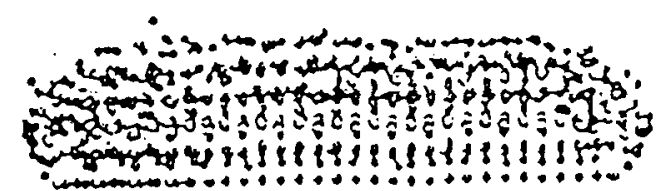

(h)

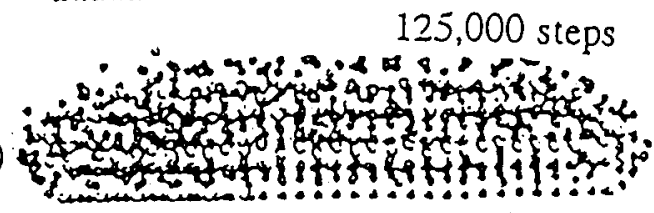

150,000 steps

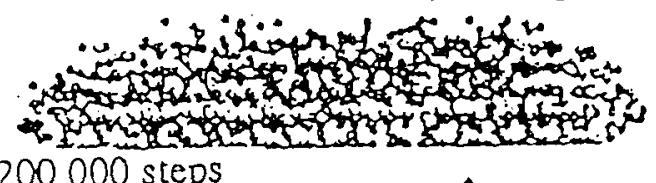

(i)

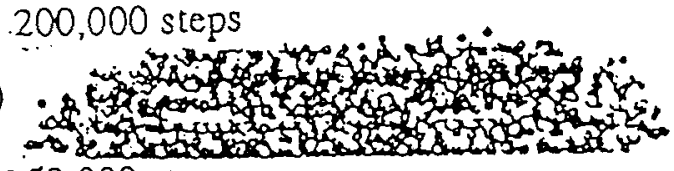

250,000 steps

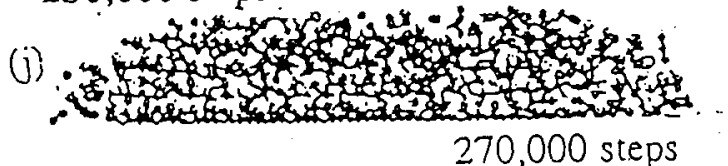

Figure 10. Atomic positions projected on ( $\overline{1} \overline{1} 2$ ) for compression. (a) start, (b) at 25,000 step, (c) at 50,000 steps, (d) at 75,000 steps, (e) at 100,000 steps, (f) at 125,000 steps, (g) at 150,000 steps, (h) at 200,000 steps, (i) at 250,000 steps, (j) at 270,000 steps.

sharp yield stress was observed. In the cutting of silicon specimens chip was always found to be amorphous and the cut surface became sometimes amorphous. The chip was recrystallized into small crystals in case of copper. It may be possible that silicon can be plastically deformed by a very high speed deformation at lower temperatures.

\section{References}

Doyama M 1997 Nanostruct. Mater. 9689
Doyama M and Kogure Y 1997 Radiat. Eff. Defects Solids 142 565

Oh D A and Johnson R A 1986 J. Mater. Res. 3471

Oh D A and Johnson R A 1989 in Atomistic simulation of materials (eds) V Vitek and D J Srolovitz (New York: Plenum Press) p. 233

Stillinger F H and Weber T A 1983 J. Phys. Chem. 87 2833

Stillinger F H and Weber T A 1984 J. Chem. Phys. 80 4434

Stillinger F H and Weber T A 1985 Phys. Rev. B31 5262 